\title{
Descripción de manifestaciones ansiosas, depresivas y autoconcepto en pacientes obesos mórbidos, sometidos a cirugía bariátrica
}

\author{
CARMEN ROJAS ${ }^{1, a}$, MARÍA BRANTE ${ }^{2, a}$, \\ EDGARDO MIRANDA ${ }^{3, \mathrm{~b}}$, RICARDO PÉREZ-LUCO ${ }^{4, \mathrm{c}}$
}

\section{Anxiety, depression and self-concept among morbid obese patients before and after bariatric surgery}

Background: There is conflicting evidence about the emotional impact of bariatric surgery among obese individuals. Aim: To evaluate anxiety, depression and self-concept, before and after bariatric surgery among patients with morbid obesity. Material and Methods: Fifteen males and 5 females aged 28 to 61 years with a mean body mass index of $42.6 \pm 5 \mathrm{~kg} / \mathrm{m}^{2}$, were studied. Grid Technique (TR), Personal Self Evaluation Questionnaire (OQ-45.2) and Anxiety Sate-Trait Inventory (STAI) were applied before and six months after bariatric surgery. Results: After surgery, the scores of self-esteem, well-being, flexibility, attraction, self-acceptance, confidence and guilt improved significantly. STAI and OQ-45.2 anxiety and depressive symptoms assessment were in the normal range before and after surgery. However, after surgery, there was a significant decrease in these sub scales in the OQ-45.2. No significant changes in anxiety measured using the STAI, were observed after surgery. Conclusions: Six months after bariatric surgery, positive changes were found in selfconcept, and overall well-being.

(Rev Med Chile 2011; 139: 571-578).

Key words: Bariatric surgery; Obesity, morbid; Self assessment.

'Instituto de Neurociencias
Clínicas, Facultad de
Medicina, Universidad
Austral de Chile.
'Universidad Santo Tomas,
Osorno.
'Facultad de Psicología,
Universidad Autónoma,
Madrid.
'Departamento de
Psicología, Universidad de
La Frontera, Temuco.
aPsicóloga, Magíster en
Psicología clínica.
bPsicólogo, Máster en
Metodología.
'Psicólogo, Doctor en
Psicología.
Recibido el 1 de julio de
2010, aceptado el 23 de
marzo de 2011.
Correspondencia a:
Carmen Rojas Carvajal,
Universidad Austral de
Chile; Campus Isla Teja s/n,
Valdivia.
Fono: 063-221327
E-mail: carmenrojas@
uach.cl

L a obesidad es una patología de alta prevalencia a nivel mundial y nacional, siendo creciente su incidencia ${ }^{1}$ y llegando a cifras preocupantes en nuestro país ${ }^{2-4}$. De acuerdo a la Encuesta Nacional de Salud en Chile, 2003 3 , 22\% de la población sufre obesidad, y 1,3\% obesidad mórbida; siendo esta última, dos veces más frecuente en mujeres. En este contexto, la obesidad mórbida (entendida antropométricamente como índice de masa corporal [IMC] igual o mayor a 40), se asocia a una serie de complicaciones médicas, como el síndrome metabólico, la diabetes tipo II y cardiopatías, entre otras ${ }^{2}$. Estos pacientes también presentan una elevada comorbilidad psiquiátrica, siendo las ma- nifestaciones más frecuentes: ansiedad, depresión y trastornos de la conducta alimentaria ${ }^{5-7}$.

Existen estudios que avalan las tendencias depresivas en la obesidad, especialmente en mujeres, así como aumento de la ansiedad ${ }^{7,8}$, autoconcepto negativo, mayor frecuencia de trastornos de alimentación y menor interacción social, relacionados directamente con su condición de obesidad ${ }^{9,10}$.

Los estudios que relacionan personalidad con obesidad mórbida no han encontrado asociación con un estilo de personalidad específico. Señalándose diversos perfiles ${ }^{11}$ con gran heterogeneidad. Algunos autores ${ }^{9}$ describen dificultades emocionales, destacando las tendencias depresivas. Por 
su parte, Chaguán (Ponencia presentada en el III Congreso IFSO Latinoamericano, 2009) encuentra en dichos pacientes, una alta represión emocional y dificultad de manifestar necesidades afectivas, así como elementos de personalidad inseguros y ansiosos.

En este contexto, algunos autores ${ }^{12,13}$ plantean que los pacientes obesos, especialmente mujeres, presentarían una relación entre sobrealimentación y alexitimia, (dificultad para identificar en sí mismo el componente afectivo de las emociones y expresarlo verbalmente). Lo anterior implicaría limitaciones para el reconocimiento y posterior modulación afectiva, especialmente de estados emocionales negativos, favoreciendo conductas de sobrealimentación, como un modo de autorregulación ${ }^{14}$. Sin embargo, también hay investigaciones que no muestran asociaciones significativas entre obesidad y alexitimia ${ }^{15}$.

En síntesis, la obesidad es un trastorno multifactorial, resultante de factores etiopatogénicos multivariados: bio-psico-sociales ${ }^{2,16,17}$. Sin embargo, los factores psicológicos están teniendo cada vez más peso, existiendo fuertes evidencias que apoyan el considerar a la obesidad como un trastorno mental y no sólo orgánico ${ }^{6,18}$.

En Chile, se han realizado tratamientos médicos multidisciplinarios, utilizando variadas alternativas: dieta, actividad física, terapia psicológica y fármacos, entre otros; los que particularmente en pacientes con obesidad mórbida, han fracasado $^{2,19}$. Frente a esto, en los últimos 10 años, la alternativa quirúrgica ha mostrado ser la opción más efectiva ${ }^{20-22}$.

La pérdida de peso producto de la cirugía bariátrica tiene consecuencias psicosociales positivas $^{23}$. Los pacientes mejoran sus índices de calidad de vida, con un incremento de la salud global y una mejor adaptación social y disminución de los síntomas ansiosos y depresivos ${ }^{7,24,25}$. En relación con la salud mental de las personas obesas post cirugía, Koppmann (Ponencia presentada en el III Congreso IFSO Latinoamericano, 2009), señala que mejoran los trastornos ansiosos y depresivos, pero no se modifican aspectos caracterológicos. Sin embargo, existen estudios que muestran evidencias que refutan esto, es decir, que no hay una asociación significativa entre obesidad, depresión $\mathrm{y}$ ansiedad ${ }^{26,27}$.

Otros estudios ${ }^{28-30}$ señalan que la disminución y mantención del peso después del tratamiento quirúrgico depende de varios factores, entre ellos, el estado psiquiátrico del paciente. En este sentido algunos autores ${ }^{6,24,29}$ sugieren realizar apoyo psiquiátrico previo y posterior a la cirugía, para promover una mayor estabilidad psicológica del paciente, favoreciendo la mantención del peso logrado.

A la luz de los contradictorios antecedentes, tanto de manifestaciones psicológicas asociadas a la obesidad mórbida, así como en relación al impacto emocional producido por la cirugía bariátrica, cabe preguntarse: ¿Cambian la ansiedad, depresión y autoconcepto en pacientes obesos mórbidos al ser sometidos a cirugía bariátrica?.

Se propuso como objetivo general: evaluar cambios en las variables de estudio, ya mencionadas, entre el momento previo a la cirugía (una semana antes) y seis meses después de ésta, en centros de salud de las ciudades de Valdivia y Puerto Varas.

Como objetivos específicos: determinar la existencia de diferencias entre los dos momentos evaluados en los 20 pacientes respecto a: a) funcionamiento ansioso (rasgo y estado) usando el Inventario de Ansiedad Estado-Rasgo (STAI); b) sintomatología ansiosa-depresiva (SD), relaciones interpersonales (RI) y rol social (RS), medidos con el OQ-45.2; y c) en autoconcepto, usando la técnica de la rejilla (TR) para evaluación de constructos personales.

\section{Método}

\section{Participantes}

Se utilizó un muestreo no probabilístico intencionado, accediendo a pacientes disponibles en las regiones de Los Ríos y Los Lagos: 15 mujeres y 5 hombres, con edades entre 28 y 61 años (Tablas 1 y 2). Todos los participantes, diagnosticados con obesidad mórbida, habían sido categorizados aptos para acceder a cirugía bariátrica, tras evaluación psicológica y psiquiátrica (previa a este estudio, realizado por profesionales de cada institución), descartándose pacientes con presencia de trastornos anímicos severos, alteraciones de personalidad (especialmente limítrofe), esquizofrenia u otra patología psicótica, abuso o dependencia de alcohol o drogas; y retardo mental.

Se resguardó la ética en la totalidad del proceso de investigación (autorización del comité de ética 
del Hospital Valdivia) y firma previa y voluntaria de consentimiento informado.

\section{Diseño}

Se realizó un estudio no experimental descriptivo, de grupo único, con mediciones antes y después (una semana previa a la operación y seis meses después de ésta).

Tabla 1. Frecuencias variables sociodemográficas

\begin{tabular}{|llrr|}
\hline Variables & Valor & n & (\%) \\
& Masculino & 5 & 25 \\
& Femenino & 15 & 75 \\
Tipo operación & Bypass gástrico & 13 & 40 \\
& Manga gástrica & 7 & 60 \\
& Valdivia & 65 \\
& Bscolaridad & 12 & 5 \\
& Medicos & 3 & 15 \\
& Medios incompletos & 5 & 25 \\
& Técnicos superiores & 6 & 30 \\
& Universitarios & 5 & 25 \\
\multirow{2}{*}{ Estado civil } & Casados & 13 & 65 \\
& Separados & 3 & 15 \\
& Convivientes & 1 & 5 \\
& Solteros & 3 & 15 \\
\hline
\end{tabular}

\section{Instrumentos}

Inventario de Ansiedad Estado-Rasgo STAI $\mathrm{E}-\mathrm{R}^{31}$ : Inventario de auto-reporte, diseñado para evaluar ansiedad como estado (STAI-E) y como rasgo (STAI-R). La puntuación total para cada dimensión oscila entre 0 y 60 , transformando puntuaciones directas en percentiles en función de sexo y edad (sin puntaje de corte). El estudio de Vera ${ }^{32}$ permitió generar normas, determinar su validez de constructo mediante análisis factorial confirmatorio y establecer la confiabilidad por consistencia interna para cada escala.

Cuestionario de Autoevaluación OQ-45.2 $2^{33}$. Instrumento de auto-reporte que mide estados y no rasgos. Consta de tres escalas: síntomas ansiosos y depresivos (SD); relaciones interpersonales (RI); y rol social (RS). Además, entrega un puntaje general que abarca las tres escalas antes señaladas. La tabulación muestra que a mayor puntaje más disconfort, con puntos de corte (PC) para la población chilena que discriminan población funcional de disfuncional (Tabla 3). El instrumento demostró ser confiable, mostrando validez concurrente con el $\mathrm{DAS}^{34}$, y validez en su sensibilidad a la psicopatología y al cambio ${ }^{35}$.

Técnica de la Rejilla $T R^{36}$ : Formulada desde la concepción constructivista de Kelly ${ }^{37}$, que concibe un ser humano activo que interpreta los sucesos de la realidad de acuerdo a sus propios significados subjetivos, expresados en constructos personales bipolares, los cuales representan distintos aspectos de su identidad. La rejilla está constituida por elementos, en este caso aspectos del sí mismo (yo

Tabla 2. Estadísticos descriptivos de la muestra por variables de interés (antes y después de la operación)

\begin{tabular}{|c|c|c|c|c|}
\hline & Media aritmética & Desviación estándar & Valor mínimo & Valor máximo \\
\hline \multicolumn{5}{|l|}{ Antes } \\
\hline Edad (años) & 44,10 & 9,92 & 28,00 & 61,00 \\
\hline Talla (m) & 1,59 & 0,07 & 1,48 & 1,75 \\
\hline Peso (kg) & 108,63 & 20,89 & 83,00 & 174,00 \\
\hline $\operatorname{IMC}\left(\mathrm{kg} / \mathrm{m}^{2}\right)$ & 42,57 & 5,05 & 37,60 & 58,80 \\
\hline \multicolumn{5}{|l|}{ Después } \\
\hline Edad (años) & 44,85 & 9,73 & 29,00 & 62,00 \\
\hline Talla (m) & 1,59 & 0,07 & 1,48 & 1,75 \\
\hline Peso (kg) & 77,49 & 17,75 & 56,00 & 134,00 \\
\hline $\operatorname{IMC}\left(\mathrm{kg} / \mathrm{m}^{2}\right)$ & 30,18 & 4,96 & 25,10 & 45,30 \\
\hline
\end{tabular}


Tabla 3. Normas de puntaje de corte (PC) en el OQ45.2

\begin{tabular}{|lcc|}
\hline Área & $\begin{array}{c}\text { Rango } \\
\text { puntajes }\end{array}$ & PC* \\
\hline OQ Total & $0-180$ & 73 \\
Sintomatología (SD) & $0-100$ & 43 \\
Relaciones interpersonales (RI) & $0-44$ & 16 \\
Rol social (RS) & $0-36$ & 14 \\
\hline
\end{tabular}

*Puntaje de Corte para discriminar a una población funcional de una disfuncional ${ }^{35}$. real, yo ideal, yo antes de la operación, y después, etc) y por constructos bipolares que permiten visualizar el autoconcepto (autoestima, control, agilidad, atractivo, entre otros). Se seleccionaron los constructos más representativos mediante prueba piloto, considerando que lo que interesaba era la comunalidad del grupo a investigar (rejilla de investigación), tabulándose con escala tipo Likert, de 1 a 7 puntos (Figura 1). Dado que la rejilla no es un test convencional, no es posible una estimación de confiabilidad, no obstante, los estudios de validez

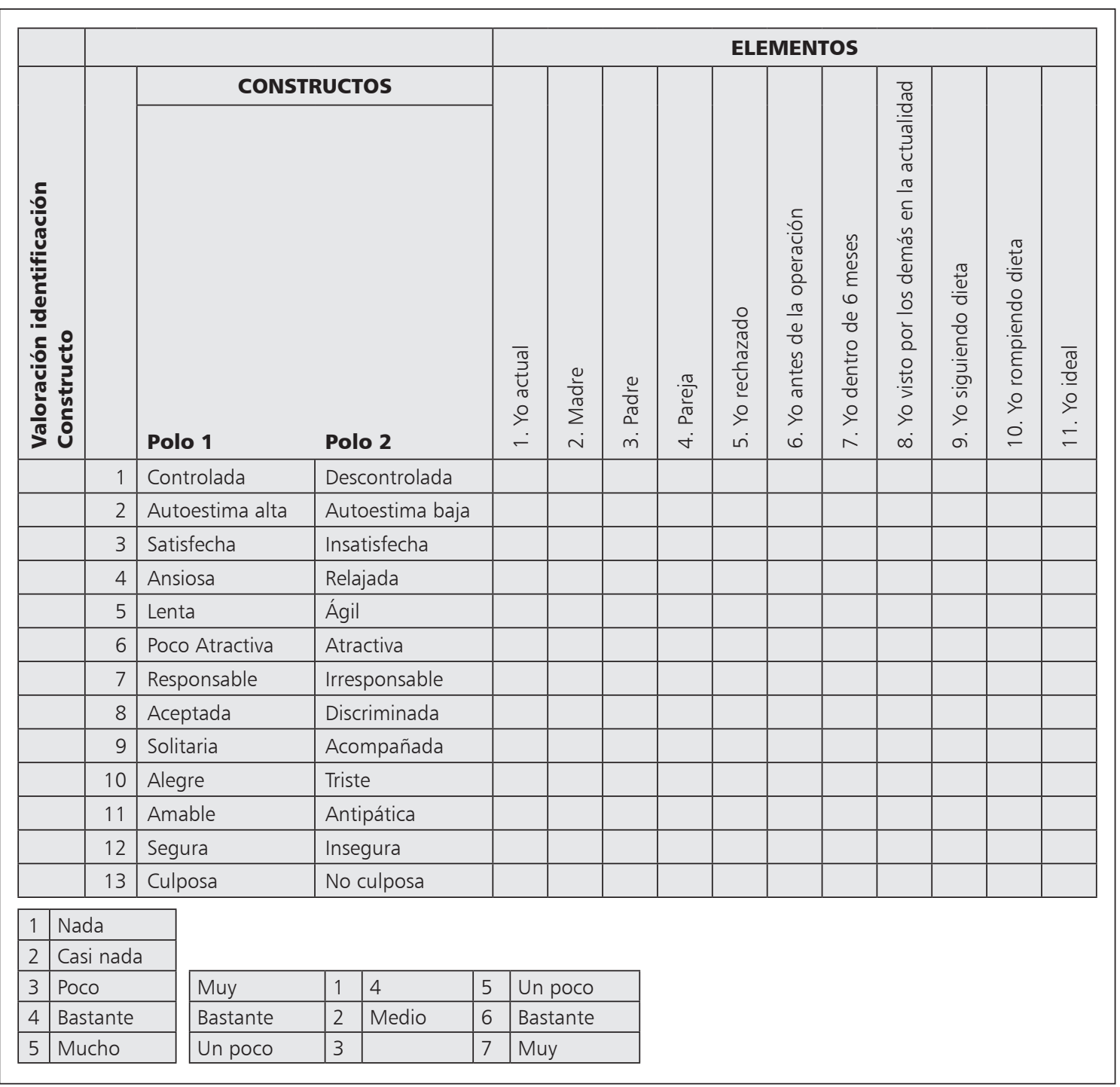

Figura 1. Modelo de Rejilla de Investigación (previa a la operación). Nota: Las respuestas se codificaron otorgando los valores más bajos (1, 2 y 3 ) al polo negativo de los constructos, y los valores más altos $(5,6$ y 7$)$ al polo positivo de los constructos. 
de constructo y predictiva arrojan resultados positivos ${ }^{36}$.

\section{Procedimiento}

La recolección de datos consistió en evaluar con los tres instrumentos de forma individual a los participantes una semana antes de la cirugía y seis meses después de ésta.

\section{Análisis de datos}

Se utilizó el software SPSS versión 15.0, para el cálculo de estadísticos descriptivos (frecuencia, porcentaje , mediana, entre otras) y comparación entre las medianas de las dos mediciones (antes/después) con los tres instrumentos (STAI, OQ-45.2 y TR) mediante la prueba $\mathrm{W}$ de Wilcoxon, por ser la que mejor se adecúa a las características de los datos (escalas de medida ordinal, distribución no normal y pertenecer a dos grupos relacionados).

\section{Resultados}

Tal como se aprecia en la Tabla 4, el STAI-E registró una reducción de 2,5 puntos entre la medición previa $(\mathrm{Md}=17,5)$ y posterior a la cirugía $(\mathrm{Md}=15)$, bajando, de acuerdo a las normas chilenas, del percentil 55 al 47. En el STAI-R la reducción fue de 7 puntos entre la medición previa $(\mathrm{Md}$ $=23,5)$ y la posterior $(\mathrm{Md}=16,5)$, disminuyendo del percentil 58 al 35 . Pese a que se aprecian diferencias en los puntajes brutos y percentiles, con una leve disminución de la ansiedad tanto de estado como rasgo, las diferencias no son estadísticamente significativas y se encuentran dentro del rango normal de ansiedad (pre y post cirugía), según normas chilenas ${ }^{32}$.

Del mismo modo, el OQ-45.2 mostró variaciones en los puntajes brutos entre las mediciones antesdespués, con medianas previas a la cirugía de 51 para OQ total; 32
Tabla 4. Medianas, puntuación z y significación estadística (p), obtenidas por los sujetos previas y posteriores a la cirugía bariátrica, con la escala de ansiedad STAI (E-R); OQ-45.2; y Técnica de la Rejilla (TR), comparadas a través de W de Wilcoxon

\begin{tabular}{|c|c|c|c|c|}
\hline & \multicolumn{2}{|c|}{ Mediana } & $\mathbf{z}$ & $\mathbf{p}$ \\
\hline \multirow[t]{2}{*}{ STAI (E) } & Antes & 17,5 & $-1,591$ & 0,112 \\
\hline & Después & 15,0 & & \\
\hline \multirow[t]{2}{*}{ STAI (R) } & Antes & 23,5 & $-1,872$ & 0,061 \\
\hline & Después & 16,5 & & \\
\hline \multirow[t]{2}{*}{ OQ-45.2 Total } & Antes & 51,0 & $-1,573$ & 0,116 \\
\hline & Después & 45,5 & & \\
\hline \multirow[t]{2}{*}{ OQ-45.2, sub-escala SD } & Antes & 32,0 & $-2,634$ & $0,008^{*}$ \\
\hline & Después & 26,0 & & \\
\hline \multirow[t]{2}{*}{ OQ-45.2, sub-escala RI } & Antes & 10,5 & $-1,432$ & 0,152 \\
\hline & Después & 12,5 & & \\
\hline \multirow[t]{2}{*}{ OQ-45.2, sub-escala RS } & Antes & 9,0 & $-0,175$ & 0,861 \\
\hline & Después & 9,5 & & \\
\hline \multirow[t]{2}{*}{ TR Controlada- Descontrolada } & Antes & 6,0 & $-0,917$ & 0,359 \\
\hline & Después & 6,0 & & \\
\hline \multirow[t]{2}{*}{ TR Autoestima Alta - Baja } & Antes & 2,0 & $-3,442$ & $0,001 * *$ \\
\hline & Después & 6,0 & & \\
\hline \multirow[t]{2}{*}{ TR Satisfecha - Insatisfecha } & Antes & 6,0 & $-2,180$ & $0,029 *$ \\
\hline & Después & 6,0 & & \\
\hline \multirow[t]{2}{*}{ TR Ansiosa - Relajada } & Antes & 3,0 & $-0,986$ & 0,324 \\
\hline & Después & 4,0 & & \\
\hline \multirow[t]{2}{*}{ TR Ágil - Lenta } & Antes & 3,0 & $-2,850$ & $0,004^{*}$ \\
\hline & Después & 6,0 & & \\
\hline \multirow[t]{2}{*}{ TR Atractiva - Poco atractiva } & Antes & 3,0 & $-2,965$ & $0,003^{*}$ \\
\hline & Después & 6,0 & & \\
\hline \multirow[t]{2}{*}{ TR Responsable - Irresponsable } & Antes & 6,0 & $-1,059$ & 0,289 \\
\hline & Después & 6,0 & & \\
\hline \multirow[t]{2}{*}{ TR Aceptada - Discriminada } & Antes & 5,5 & $-2,998$ & $0,003^{*}$ \\
\hline & Después & 6,0 & & \\
\hline \multirow[t]{2}{*}{ TR Solitaria - Acompañada } & Antes & 5,5 & $-0,756$ & 0,450 \\
\hline & Después & 6,0 & & \\
\hline \multirow[t]{2}{*}{ TR Alegre - Triste } & Antes & 6,0 & $-0,103$ & 0,918 \\
\hline & Después & 6,0 & & \\
\hline \multirow[t]{2}{*}{ TR Amable - Antipática } & Antes & 6,0 & $-0,221$ & 0,825 \\
\hline & Después & 6,0 & & \\
\hline \multirow[t]{2}{*}{ TR Segura - Insegura } & Antes & 3,0 & $-2,840$ & $0,005^{*}$ \\
\hline & Después & 6,0 & & \\
\hline \multirow[t]{2}{*}{ TR Culposa - No culposa } & Antes & 2,5 & $-2,139$ & $0,032 *$ \\
\hline & Después & 5,5 & & \\
\hline
\end{tabular}

${ }^{*} p \leq 0,05 ;{ }^{*} p \leq 0,001$ 
para SD; 10,5 para RI y 9 para RS. En la medición posterior a la cirugía, se observó una disminución tanto del OQ total, como de la sub-escala SD, con medianas de 45,5 y 26 respectivamente. En cuanto a la sub escala RI, esta aumentó, al igual que RS con medianas de 12,5 y 9,5 puntos, respectivamente. Sólo se evidencian diferencias significativas en la sub-escala SD $(\mathrm{p}<0,05)$, lo que implica una disminución significativa de los síntomas ansiosos y depresivos posteriores a la cirugía (Tabla 4). Cabe señalar que aunque existen diferencias estadísticamente significativas entre los puntajes previos y posteriores en la sub-escala $\mathrm{SD}$, ambos puntajes se encontraron dentro de rangos normales (población sin disfuncionalidad) de acuerdo a las normas chilenas y la variación no sería clínicamente significativa ${ }^{35}$.

En la evaluación con la TR, en el autoconcepto, se evidenció que el constructo autoestima alta v/s autoestima baja presentó el mayor incremento $(\mathrm{p}=0,001)$, al considerar el yo actual, antes y después de la cirugía. Asimismo, los participantes señalaron que a los seis meses posteriores a la cirugía experimentaron mayores niveles de satisfacción $(\mathrm{p}=0,029)$, agilidad $(\mathrm{p}=0,004)$, atractivo $(\mathrm{p}=0,003)$, aceptación $(\mathrm{p}=0,003)$, seguridad $(\mathrm{p}=0,005)$ y disminución del sentimiento de culpa $(\mathrm{p}=0,032)$. El resto de los constructos no mostró cambios significativos (Tabla 4).

\section{Discusión}

Los resultados obtenidos en relación a los constructos del autoconcepto, (TR) son coherentes con la literatura antes señalada ${ }^{7,23,25}$, así poco más de la mitad (7 de 13), se incrementan significativamente a los seis meses después de la cirugía bariátrica. Se evidencia en los pacientes una autopercepción más positiva, con un importante aumento en la autoestima. Además se sienten más atractivos, más satisfechos, más ágiles, más aceptados, con mayor seguridad y menos culpas. El resto de los constructos no varió significativamente.

Por otro lado, no se observan cambios significativos en los niveles de ansiedad reportados por el STAI. Ello es esperable, considerando que la evidencia científica disponible, que relaciona obesidad con trastornos ansiosos, es contradictoria $^{23,24,26,27}$.

Sin embargo, aparece una disminución significativa en la sub-escala de síntomas ansiosos y depresivos (SD) del OQ-45.2 después de la cirugía, lo cual es concordante con lo planteado en la mayor parte de la literatura ${ }^{23-25}$, especialmente en los primeros 6 meses posteriores, que es donde la baja de peso es mayor, experimentando mayores sensaciones de satisfacción y escasas frustraciones. En relación a las sub-escalas RI y RS, así como en el OQ total, no hay diferencias significativas. Hay que considerar que desde antes de la operación los pacientes reportan puntajes, (ansiedad y depresión) que los ubican dentro de la población sin disfuncionalidad, pero posterior a la cirugía experimentan un mayor confort.

Una hipótesis explicativa de los hallazgos considera lo planteado por la literatura ${ }^{7,12,14}$ sobre las dificultades de los pacientes con obesidad para contactarse adecuadamente con sus emociones (alexitimia) y sus dificultades en el área de las relaciones interpersonales y rol social (área laboral); lo que les hace minimizar estos problemas y sufrimientos o simplemente no expresarlos frente a un contexto de evaluación, particularmente cuando se usan instrumentos de autoreporte, en este sentido resultaría interesante usar medidas de observación externa (fisiológicas y conductuales) para la ansiedad.

Tomando en consideración, los antecedentes ya señalados, es posible plantear que el tema de la obesidad es muy complejo de abordar, teniendo en cuenta su gran variabilidad, pues no existe un solo tipo de obeso. Al parecer las dificultades para estandarizar resultados se deben a: la utilización de muestras pequeñas, diferentes etiologías de la obesidad, técnicas quirúrgicas diversas, historia personal y familiar de obesidad y métodos para medir las variables; además de escasas investigaciones pre y post cirugía, falta de seguimiento a largo plazo, y gran variedad en los criterios, particularmente de lo que se entiende por éxito de la intervención.

El presente estudio, evidencia alguna de las limitaciones metodológicas antes señaladas: muestra pequeña, ausencia de grupo control, existencia de comorbilidades orgánicas o psiquiátricas (especialmente características de personalidad), uso de medicamentos, entre otros. Sin embargo, parecen no existir a la fecha investigaciones de seguimiento psicológico pre y post cirugía, consignados en alguna publicación científica en Chile, por ello, este trabajo es un aporte preliminar para el inicio de una línea tanto de investigación como 
de intervención psicoterapéutica de las variables psicológicas que impactan los resultados de la cirugía bariátrica,

Por tanto, parece imprescindible desarrollar estudios con seguimiento a largo plazo ( 3 a 5 años), con muestras más representativas y con instrumentos distintos al autoreporte, que permitan resolver el tema de la deseabilidad social y de la probable dificultad de los pacientes para reconocer y expresar estados emocionales.

Otras áreas a estudiar son: definir predictores de éxito o fracaso, diferenciar los distintos perfiles que existen dentro de la obesidad mórbida (trastornos de personalidad, estructuras ansiosas, depresivas, estructuras adictivas, entre otras). Evaluando en forma diferencial, cada uno de estos grupos y unificando el tipo de cirugía.

En futuras líneas de intervención, a nivel psicoterapéutico, sería importante tomar en cuenta la información obtenida en la evaluación prequirúrgica, para diseñar y evaluar en el largo plazo programas específicos para las necesidades de cada paciente, con un apoyo que permita mantener en el tiempo los cambios (particularmente en el autoconcepto) asociados a los resultados de la cirugía. Éstos debieran considerar los predictores de éxito y fracaso y las características individuales para usarlos como plataforma desde donde producir los cambios, inicialmente en un mejor manejo del control de impulsos y tolerancia a la frustración, y luego en mayor regulación y autoconciencia emocional, para contribuir finalmente a la estabilidad y mantención de esta nueva construcción del sí mismo, sin obesidad.

A modo de conclusión, se aprecian a corto plazo cambios muy positivos, especialmente en el autoconcepto y estado anímico de los pacientes, post cirugía. Sin embargo, considerando que la obesidad es un problema de salud pública en Chile, es necesario aislar características emocionales y de personalidad, que a largo plazo, permitan predecir la evolución post quirúrgica y realizar intervenciones, especialmente a nivel psicoterapéutico, más eficaces. Lo anterior, aminoraría costos asociados a los fracasos quirúrgicos, siendo de gran impacto en la evolución de pacientes que ingresan a los programas de obesidad mórbida, implementados en el sistema público de salud.

Agradecimientos: Los autores agradecemos al Mg. en Psicología José Luis Molina, por su permanente apoyo y colaboración, asimismo a las psicólogas Alejandra Fritis, Claudia Sutulov y a las Mg. en Psicología Daniela Zúñiga y Claudia Lucero, además al Dr. Jaime Silva Concha, a los psiquiatras Edwin Krogh, y Alejandro Koppmann, a los médicos cirujanos Dr. Carlos Cárcamo y Felix Raimann, y a la EU. Claudia Chandia. También al Hospital Base Valdivia, y Clínica Alemana de Puerto Varas, así como a todos los pacientes que participaron en este estudio, anónima y desinteresadamente.

\section{Referencias}

1. Vio F, Salinas J. Promoción y calidad de vida en Chile: una política con nuevos desafíos. Rev Chil Nutr 2006; 33: 252-9.

2. Cuevas A, Reyes MS. Lo último en diagnóstico y tratamiento de la obesidad. ¿Hay lugar aún para la terapia conservadora?. Rev Med Chile 2005; 133: 713-22.

3. Pontificia Universidad Católica de Chile, Facultad de Medicina, Departamento de Salud Pública. 2003. Encuesta Nacional de Salud (ENS). http://escuela.med.puc. cl. [Consultado el 21 de agosto, 2008].

4 Ministerio de Salud de Chile. Estudio de Carga de Enfermedad y Carga Atribuible, 2008. http://epi.minsal.cl. [Consultado el 10 de septiembre, 2009].

5. Black D, Goldstein R, Mason E. Psychiatric diagnosis and weight loss following gastric surgery for obesity. Obes Surg 2003; 13: 746-51.

6. Bustamante F, Williams C, Vega E, Prieto B. Aspectos psiquiátricos relacionados con la cirugía bariátrica. Rev Chil Cir 2006; 58: 481-5.

7. Ríos B, Rangel G, Álvarez R, Castillo F, Ramírez G, Pantoja J, et al. Ansiedad, depresión y calidad de vida en el paciente obeso. Acta Med Gpo Ang 2008; 6: 147-53.

8. Onyike C, Crum R, Lee H, Lyketsos C, Eaton W. Is obesity associated with major depression? Results from the Third national health and nutrition examination survey. Am J Epidemiol 2003; 158: 1139-47.

9. Elfhag K, Carlsson AM, Rössner S. Subgrouping in obesity based on Rorschach personality characteristics. Scand J Psychol 2003; 44: 399-407.

10. Puhl R, Heuer C. The stigma of obesity: A review and update. Obes Res 2009; 17: 941-964.

11. Van Hout GC, Van Oudheusden I, Krasuska AT, Van Heck GL. Psychological profile of candidates for vertical banded gastroplasty. Obes Surg 2006; 16: 67-74.

12. De Chouly De Lenclave M-B, Florequin C, Bailly D. Obesity, alexithymia, psychopathological disorders, and binge eating: a comparative study between 40 obese 
subjects and 32 controls. L'Encéphale 2001; 27: 343-50.

13. Pinaquy S, Chabrol H, Simon C, Louvet J-P, Barbe P. Emotional eating, alexithymia, and binge-eating disorder in obese women. Obes Res 2003; 11: 195-201.

14. Silva J. Sobrealimentación Inducida por la Ansiedad, Parte II: Un Marco de Referencia Neurocientífico para el Desarrollo de Técnicas Psicoterapéuticas y Programas de Prevención. Terapia Psicológica 2008; 26: 99-115.

15. Adami G, Campostano A, Ravera G, Leggieri M, Scopinaro N. Alexithymia and body weight in obese patients. Behav Med 2001; 27: 121-6.

16. Cabello M, Zúñiga J. Aspectos intrapersonales y familiares asociados a la obesidad. Ciencia UANL 2007; 2: 183-8.

17. Chinchilla A. Obesidad y psiquiatría. En Quintero FJ, Correas J, García V, Chinchilla, A, Editors, Concepto y Clasificación de la Obesidad. Barcelona, España: Editorial Masson; 2005. p. 9-14.

18. Cordella P. ¿Incluir la obesidad en el manual de enfermedades mentales (DSM IV)?. Rev Chil Nutr 2008; 35: 181-7.

19. Guisado JA, Vaz F J. Características clínicas de los pacientes con obesidad mórbida. Rev Psiquiatría Fac Med Barna 2002; 29: 85-94.

20. Carrasco F, Klaassen J, Papapietro K, Reyes E, Rodríguez L, Csendes A, et al. Propuesta y fundamentos para una norma de manejo quirúrgico del paciente obeso. Año 2004. Rev Med Chile 2005; 133: 699-706.

21. Hamilton J. Resultados a 20 meses de la banda gástrica ajustable en el tratamiento de la obesidad mórbida. Rev Chil Cir 2004; 56: 307-16.

22. Csendes A, Burdiles P, Papapietro K, Burgos AM. Comparación del tratamiento médico y quirúrgico en pacientes con obesidad grado III (obesidad mórbida). Rev Med Chile 2009; 137: 559-66.

23. Dymek M, Le Grange D, Neven K, Alverdy J. Quality of life and psychosocial adjustment in patients after rouxen-y gastric bypass: a brief report. Obes Surg 2001; 11: 32-9.

24. Guisado JA, Vaz FJ, Rubio MA. Diferencias psicológicas en pacientes con obesidad mórbida tras cirugía gástrica. Rev Psiquiatría Fac Med Barna 2002; 29: 213-7.

25. Barreto N, Braghrolli O, Lima K, Eduarda B, Seal C, Santos D. Quality of life of obese patients submitted to bariatric surgery. Nutr Hosp 2004; XIX: 367-71.

26. Cassinello N, Fernández R, Álvarez S, Baguena M, Ortega J. Valoración de la imagen corporal y cambios de expectativas en los pacientes sometidos a by-pass gástrico. Nutr Hosp 2007; 22: 254-67.

27. Rivenes A, Harvey S, Mykletun A. The relationship between abdominal fat, obesity, and common mental disorders: Results from the HUNT Study. J Psychosom Res 2009; 66: 269-75.

28. Clark M, Balsiger B, Sletten C, Dahlman K, Ames G, Williams D, et al. Psychosocial factors and 2-year outcome following bariatric surgery for weigth loss. Obes Surg 2003; 13: 739-45.

29. Kinzl J F, Trefalt E, Fiala M, Biebl W. Psychotherapeutic treatment of morbidly obese patients after gastric banding. Obes Surg 2002; 12: 292-4.

30. Byrne S, Cooper Z, Fairburn C. Weight maintenance and relapse in obesity: a qualitative study. Int J Obes 2003; 27: 955-62.

31. Spielberger CD, Gorsuch RL, Lushene RE. Manual for the State-Trait Anxiety Inventory. Palo Alto, California: Consulting Psychologists Press; 1970.

32. Vera P, Celis K, Cordova N, Buela G, Spielberger CD. Preliminary Analysis and Normative Data of the StateTrait Anxiety Inventory (STAI) in Adolescent and Adults of Santiago, Chile. Terapia Psicológica 2007; 25: 155-62.

33. Lambert MJ, Hansen NB, Umpress V, Lunnen K, Okiishi J, Burlingame GM. Administration and Scoring Manual for the OQ-45.2. Brigham, USA: American Professional Credentialing Services LLC; 1996.

34. Alvarado R, Vera A. Validación de Escala para medir Trastornos Depresivos. Por Ansiedad y Somatomorfos (DAS). Santiago de Chile, Cuadernos de Trabajo No 2. Universidad Diego Portales; 1991

35. De La Parra G, Von Bergen A. OQ -45.2, Cuestionario para evaluación de resultados y evolución en psicoterapia: Adaptación, validación e indicaciones para su aplicación e interpretación. Terapia Psicológica 2002; 20: 161-76.

36. Feixas G, Cornejo JM. Manual de la técnica de rejilla mediante el programa RECORD 2.0.2nd ed. Barcelona: Paidós; 1996.

37. Kelly G. The Psychology of Personal Constructs. New York: Norton; 1955. 